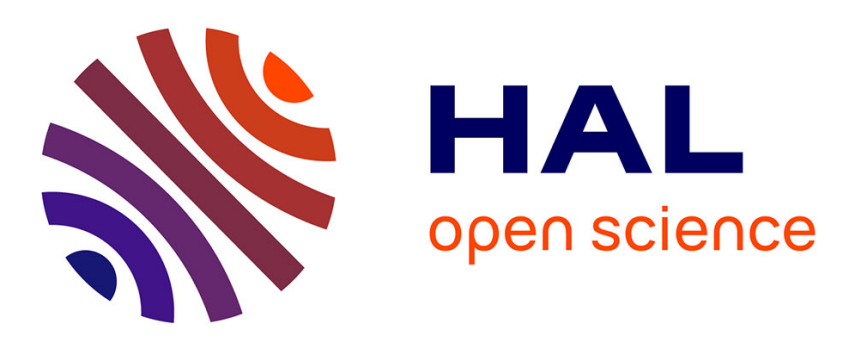

\title{
Influence of a hysteretic damper on the flutter instability
} Arnaud Malher, Olivier Doaré, Cyril Touzé

\section{To cite this version:}

Arnaud Malher, Olivier Doaré, Cyril Touzé. Influence of a hysteretic damper on the flutter instability. Journal of Fluids and Structures, 2017, 68, pp.356 - 369. 10.1016/j.jfluidstructs.2016.11.001 . hal01442349

\section{HAL Id: hal-01442349 \\ https://hal.science/hal-01442349}

Submitted on 20 Jan 2017

HAL is a multi-disciplinary open access archive for the deposit and dissemination of scientific research documents, whether they are published or not. The documents may come from teaching and research institutions in France or abroad, or from public or private research centers.
L'archive ouverte pluridisciplinaire HAL, est destinée au dépôt et à la diffusion de documents scientifiques de niveau recherche, publiés ou non, émanant des établissements d'enseignement et de recherche français ou étrangers, des laboratoires publics ou privés. 


\title{
Influence of a hysteretic damper on the flutter instability
}

\author{
Arnaud Malher, Olivier Doaré, Cyril Touzé \\ IMSIA (Institute of Mechanical Sciences and Industrial Applications), \\ ENSTA ParisTech - CNRS - EDF - CEA, \\ Université Paris-Saclay, \\ 828 Bd des Maréchaux, 91732 Palaiseau Cedex, France
}

\begin{abstract}
The influence of a hysteretic damper on the airfoil flutter instability is investigated. In particular, its effect on the post-critical limit cycle oscillations (LCOs) is emphasized. For that purpose, an aeroelastic model including large amplitude motions and dynamic stall phenomenon, is considered for a rigid flat plate having two degrees of freedom in pitch and plunge motions. The hysteretic behaviour is modeled thanks to a generalized Bouc-Wen formulation. A parametric study of aeroelastic as well as hysteresis model parameters, allows one to draw a complete picture of the bifurcation scenario, highlighting the capacity of the hysteretic damper in precluding the occurrence of stall. The special case of shape memory alloy (SMA) springs is then used numerically and experimentally for controlling the flutter oscillations of a flat plate. The study reveals the ability of the SMA springs to drastically reduce the amplitudes of the LCOs caused by the flutter instability. Keywords: Flutter instability, dynamic stall, hysteretic damper, shape memory alloys
\end{abstract}

\section{Introduction}

Undesired mechanical vibrations are a major issue in many industrial applications. Multiple strategies exist to avoid them, depending on the type of vibration or the expecting operating range. This paper focuses on the control of the airfoil classical flutter instability. This phenomenon results from an interaction between two modes of the structure and an axial flow, which gives rise to strong or even fatal deformations. The flow velocity for which the instability arises is called flutter velocity. The goal of the control is then to increase the flutter velocity and reduce the amplitude of vibrations developing in the post-critical regime.

Flutter control is an important research topic in aeronautics. In the last decades, the most explored strategy to prevent flutter was to actively control the unsteady aerodynamic loads on the airfoil,

Email address: arnaud.malher@ensta-paristech.fr (Arnaud Malher)

January 9, 2017 
see e.g. Dowell (2004), Karpel (1982), Ko et al. (1999), and Vipperman et al. (1998). Nevertheless some studies have investigated passive control strategies, like the implementation of a nonlinear energy sink by Y. S. Lee et al. (2007a,b) acting on both modes of the wing or a tuned mass damper by Kwon and Park (2004) acting on a bridge deck.

In the present study, the passive control is realized by means of a hysteretic damper which consists of springs made of shape memory alloys (SMA) acting on the plunge motion. Indeed, in their pseudo-elastic regime, SMA are known to dissipate an important amount of energy due to the presence of an hysteresis loop in their stress-strain relationship, see e.g. Doaré et al. (2012) and Ould Moussa et al. (2012).

Carboni and Lacarbonara (2015) and Carpineto et al. (2014) have studied numerically and experimentally the dynamical behaviour of hysteretic damper partly made of SMA wires. These studies have shown promising results regarding the capacity of such damper to mitigate unwanted vibrations.

Besides the study of the SMA hysteretic behaviour, a particular attention is paid in this work on the estimation of the aerodynamic loads developing around the airfoil. Indeed, in the post-critical regime, the airfoil is subjected to large amplitude motions and a nonlinear approach must be considered to estimate the aerodynamic forces. Especially a phenomenon called dynamic stall arises for large amplitude motion as remarked by Amandolèse et al. (2013) or Razak et al. (2011). This phenomenon is estimated by means of a phenomenological model using experiments.

The use of hysteretic damper to mitigate the flutter instability has already been explored in previous studies. For example, Candido de Sousa and De Marqui Junior (2014) considered SMA springs acting on the airfoil pitch motion. They used a refined material modeling of the SMA behaviour in order to express the restoring force. However a linear model was used to describe the aerodynamic loads. Another investigation by Lacarbonara and Cetraro (2011) also used a hysteretic damper, however it was implemented as a vibration absorber, which means that an additional degree of freedom was added to the system. Preliminary results have also been reported by Malher et al. (2015a,b). In this case, the SMA restoring force was estimated with a simple heuristic model, and the nonlinearity of the aeroelastic system by using cubic stiffness. The results presented in this study thus extend all the existing studies on the subject, by considering realistic aerodynamic nonlinear loads together with a versatile expression of the hysteretic damper, which is estimated by a generalized Bouc-Wen model.

The paper is organized as follows. In section 2, the structural model used to describe the airfoil motion along with the aerodynamic forces estimation is presented. Then, in section 3 , the modeling of the dynamic restoring force of the SMA springs is established. In section 4, an experimental set-up inspired from Amandolèse et al. (2013) is used to investigate the influence of the SMA springs on the flutter instability. Eventually, in section 5, the complete numerical model is used to explore the influence of the aerodynamic loads and the SMA springs on the flutter instability. 


\section{Airfoil model and dynamic stall characterization.}

In this section, the equation of the aeroelastic system as well as the aerodynamic loads are established. A particular attention is paid to the nonlinear behaviour of aerodynamic forces, in order to derive a predictive model able to take into account dynamic stall.

\subsection{Aeroelastic model}

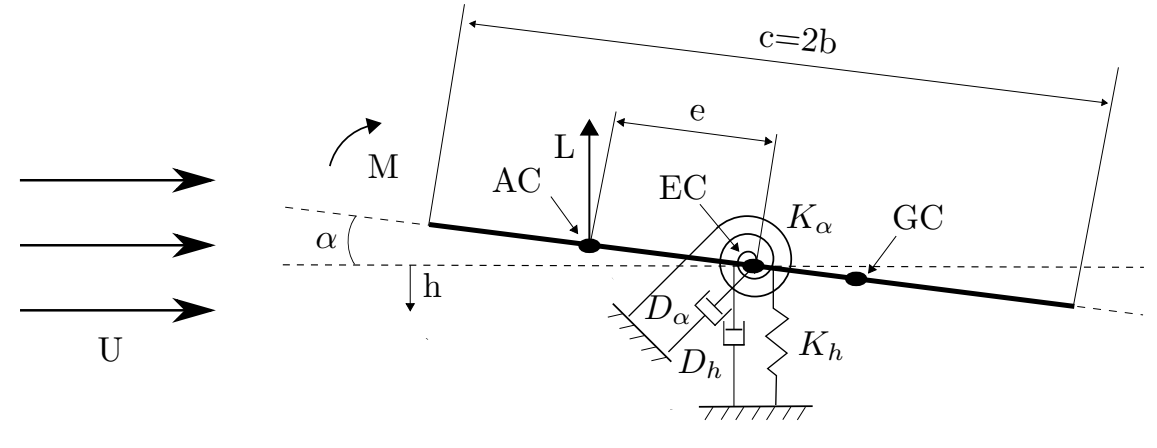

Figure 1: Two degrees of freedom flat plate section model.

The classical pitch and plunge model is used to describe the airfoil motion (see e.g Dowell (2004) for more details). The plunge (resp. pitch) motion depicts the first flexural (resp. torsional) mode of the airfoil. Pitch and plunge are respectively described by the heave $h$ and the angle of attack $\alpha$ as shown in Fig. 1, where EC refers to the elastic center, AC to the aerodynamic center and GC to the gravity center. $U$ is the upstream flow speed, $c$ the chord and $b$ the mid-chord of the plate. The distance between $\mathrm{AC}$ and EC is denoted by $e . K_{h}$ and $D_{h}$ (resp. $K_{\alpha}$ and $D_{\alpha}$ ) refer to the plunge (resp. pitch) stiffness and viscous damping. The inertia moment is denoted by $I_{\alpha}$, the airfoil mass by $m$ and the static moment by $S_{\alpha}$. The static moment is proportional to the distance between GC and EC and is responsible of the coupling. The kinetic energy reads $\mathcal{T}=\frac{1}{2} m \dot{h}^{2}+\frac{1}{2} I_{\alpha} \dot{\alpha}^{2}+S_{\alpha} \cos (\alpha) \dot{h} \dot{\alpha}$, and the potential energy which comes from the pitch and plunge stiffnesses reads $\mathcal{V}=\frac{1}{2} K_{\alpha} \alpha^{2}+\frac{1}{2} K_{h} h^{2}$. By means of a Lagrangian formulation, the equations of motion writes :

$$
\left[\begin{array}{cc}
m & S_{\alpha} \cos (\alpha) \\
S_{\alpha} \cos (\alpha) & I_{\alpha}
\end{array}\right]\left[\begin{array}{c}
\ddot{h} \\
\ddot{\alpha}
\end{array}\right]+\left[\begin{array}{cc}
D_{h} & 0 \\
0 & D_{\alpha}
\end{array}\right]\left[\begin{array}{c}
\dot{h} \\
\dot{\alpha}
\end{array}\right]+\left[\begin{array}{cc}
K_{h} & 0 \\
0 & K_{\alpha}
\end{array}\right]\left[\begin{array}{c}
h \\
\alpha
\end{array}\right]=\left[\begin{array}{c}
-L+S_{\alpha} \sin (\alpha) \dot{\alpha}^{2} \\
M
\end{array}\right]
$$

where $\left({ }^{*}\right)$ denotes the time derivative. In equation (1), $L$ is the lift and $M$ the aerodynamic moment. The detailed expressions used for $L$ and $M$, taking into account dynamic stall, are examined in the next section. 


\subsection{Dynamic Stall}

For small amplitudes, $L$ and $M$ are assumed to depend directly on an equivalent unsteady angle of attack $\alpha+\dot{h} / U$ (see e.g. Dowell (2004)). However as we are interested in the post-critical behaviour of the wing, the airfoil may encounter large amplitude motions. Thus nonlinearities have to be taken into account in the aerodynamic forces. Whereas a structural polynomial nonlinearity is generally added to the 2 dofs model flutter in order to describe the post-critical behaviour as in B. H. K. Lee et al. (1997) and Y. S. Lee et al. (2007a), the aerodynamic nonlinear forces arising from the dynamic stall phenomenon, are here taken into account. This choice is motivated by the fact that experimental results (shown later in section 4) led on a flat plate show limit cycle oscillation (LCOs) with large amplitude, around 40 degrees in pitch, in the post-critical regime. Hence dynamic stall, which appears for stall angles around 15 degrees for a NACA0012 and around 9 degrees for a flat plate, plays a significant role for the LCOs characteristics in the post-critical regime. Several models have been proposed in the literature to reproduce dynamic stall, the most frequently used are the Beddoes-Leishmann model (see Leishman and Beddoes (1989)) and the ONERA model introduced by Petot (1989). The first one endeavors to model the different steps of the dynamic stall : leading edge vortex built-up, vortex shedding, trailing edge vortex and leading edge vortex collapse. It has the advantage to be close to the physical phenomena but at the expense of a rather tedious formulation. The second one, which has been selected for the present study, is based on a phenomenological approach and relies on the use of a stall parameter extrapolated from the airfoil static lift curve. Its main advantage is to be able to fit a large variety of airfoils and flow conditions. On the other hand, it does not rely on a precise description of the physical mechanisms involved, as is usual with phenomenological models.

Let us now detail the main equations of the ONERA model (Petot (1989)). The expression of the global lift is modeled as

$$
L=\frac{1}{2} \rho S U^{2}\left(C_{l 1}+C_{l 2}\right)
$$

where $\rho$ is the fluid density, $S$ the lifting surface and $U$ the flow speed. The main variables are $C_{l 1}$, the lift unstall part, and $C_{l 2}$, the lift stalled part. Their evolutions are estimated by means of the following differential equations :

$$
\begin{aligned}
\dot{C}_{l 1}+\lambda^{L} C_{l 1} & =\lambda^{L}\left(\left.\frac{\partial C_{l}}{\partial \alpha}\right|_{\alpha=0} W_{0}+\sigma^{L} W_{1}\right)+\left(\left.\kappa^{L} \frac{\partial C_{l}}{\partial \alpha}\right|_{\alpha=0}+d^{L}\right) \dot{W}_{0}+\kappa^{L} \sigma^{L} \dot{W}_{1}, \\
\ddot{C}_{l 2}+a^{L} \dot{C}_{l 2}+r^{L} C_{l 2} & =-\left(\left.r^{L} \Delta C_{l}\right|_{W_{0}}+E^{L} \dot{W}_{0}\right) .
\end{aligned}
$$

The nonlinearity only relies on the stall parameter $\Delta C_{l}$, which is the key component of the ONERA model. This parameter is equal to the difference between the static lift extrapolated in the stall region and the actual static lift of the airfoil as shown in Fig. 2. The numerous parameters 
appearing in equation (3) may be gathered in three categories,

- the constant parameters : $\lambda^{L}$ and $\kappa^{L}$,

- the parameters showing a constant part plus a dependence on $\left(\Delta C_{l}\right)^{2}: r^{L}=r_{0}^{L}+r_{2}^{L}\left(\Delta C_{l}\right)^{2}$; $a^{L}=a_{0}^{L}+a_{2}^{L}\left(\Delta C_{l}\right)^{2}$ and $\sigma^{L}=\sigma_{0}^{L}+\sigma_{2}^{L}\left(\Delta C_{l}\right)^{2}$,

- the parameters depending only on $\Delta C_{l}$ with no constant terms : $E^{L}=-E_{2}^{L}\left(\Delta C_{l}\right)^{2}$ and $d^{L}=\sigma_{2}\left|\Delta C_{l}\right|$.

$W_{0}$ and $W_{1}$ from equation (3) are equal to $\alpha+\dot{h} / U$ and $b \dot{\alpha}$ respectively. Eventually $\left.\Delta C_{l}\right|_{W_{0}}$ is the stall parameter corresponding to the apparent angle of attack $W_{0}$.

The same model is used to estimate the aerodynamic moment, which quantities are denoted with the superscript ${ }^{M}$ instead of ${ }^{L} . \Delta C_{l}$ is estimated using static lift measurement. Eventually, $\lambda^{L}$, $\kappa^{L}, r_{0}^{L}, r_{2}^{L}, a_{0}^{L}, a_{2}^{L}, \sigma_{0}^{L}, \sigma_{2}^{L}$ and $E_{2}^{L}$ have to be fitted by means of experiments. An example of this procedure is shown in the next section for a flat plate.

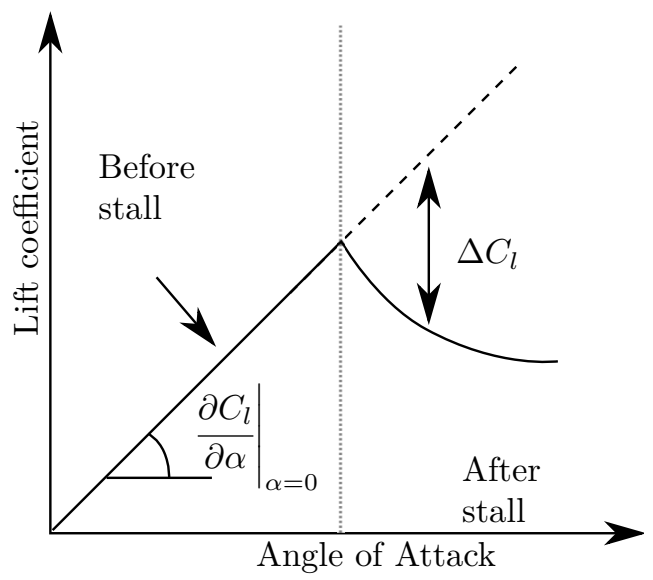

Figure 2: Schematic representation of the lift coefficient as function of the angle of attack. Solid line : static measurement. Dotted line : extrapolation of the lift coefficient before stall. The stall parameter $\Delta C_{l}$ is defined as the difference between this extrapolated line and the lift coefficient when the wing encounters stall (Petot (1989)).

\subsection{Dynamic stall measurements on a flat plate}

The goal of the present section is to measure the unsteady force and moment encountered by a flat plate controlled in pitch motion for a given range of airflow speed, pitch oscillation frequency and amplitude. The experiments are realized with a flat plate, which is the profile that will be used in the flutter experiment. The plate is rigid, rectangular and made of steel, with a span 
of $236 \mathrm{~mm}$, a chord of $70 \mathrm{~mm}$ and a thickness of $3 \mathrm{~mm}$ which means a $4.3 \%$ thickness-to-chord ratio. The plate is mounted vertically in the test-section of a closed-loop wind tunnel of width and height $0.46 \mathrm{~m}$. End plates made of plexiglass are also used. The one at the top of the profile is fixed to it and the one at the bottom is fixed to the wind tunnel test-section. A picture of the experimental set-up is given in Fig. 3. The motion is imposed by a motor controlled in amplitude and frequency. Clamped inside the motor, an aerodynamic balance, on which the plate is attached at its mid-chord, measures the normal and tangential forces applied to the profile along with the moment around the center of the balance. Acquisition and control is realized on a National Instrument acquisition board with a sampling frequency at $2 \mathrm{kHz}$. The angle of attack of the profile is measured using the feedback of the motor controller. Each acquisition is done for 25 periods of pitching oscillation.

In order to obtain precise and reliable measurements, one has to take care of two effects. The first one is the fundamental vibration mode of the plate. In our case, its eigenfrequency has been measured around $19 \mathrm{~Hz}$. Therefore, a low-pass 8th order Butterworth numeric filter with a $18 \mathrm{~Hz}$ cutoff frequency has been used for the data post-treatment. Since the largest pitch frequency tested is equal to $2 \mathrm{~Hz}$, in the non-linear regime, up to nine harmonics are taken into account, which has been found to be enough. The second effect is related to the fact that the aerodynamic balance measures all the forces, including inertia. Our measurements clearly demonstrates that this effect has to be removed from the moment, whereas it is negligible on normal and tangential force measurements. The different steps of the unsteady aerodynamics loads measurements are then :

1. set a sinusoidal pitch motion at a given frequency and amplitude using the motor,

2. get the force and moment signal by mean of the aerodynamic balance,

3. low pass filtering,

4. averaging measurement over 25 periods,

5. subtract the mean moment measured without wind to the moment measured with wind.

These measurements are exploited to fit the several parameters of equation (3). With this aim in view, the Matlab function fmincon is used, which implements a nonlinear optimization procedure. The objective function to minimize is the sum of the squared difference between measurements and model predictions. An example of the obtained results is presented in Fig. 4, where measurements of lift and aerodynamic moment at several amplitudes are plotted with the result of the ONERA model with parameters from Table 1. One can observe the good agreement between model and experiments. This procedure has been done for the several flow speed tested in the previous experiment. It has shown the ability of the ONERA model to reproduce different situations. 


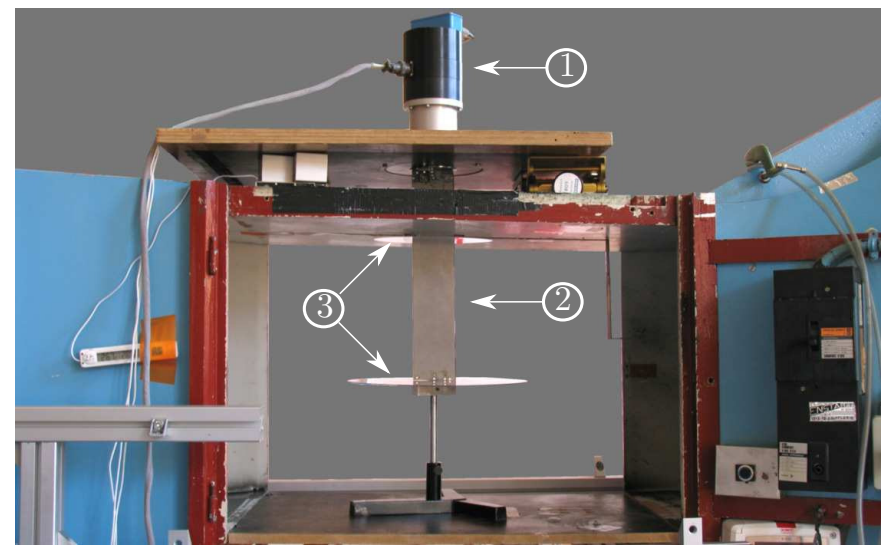

Figure 3: Dynamic stall measurement set-up. 1 : Motor with aerodynamic balance inside, 2 : flat plate and 3 : end plates.

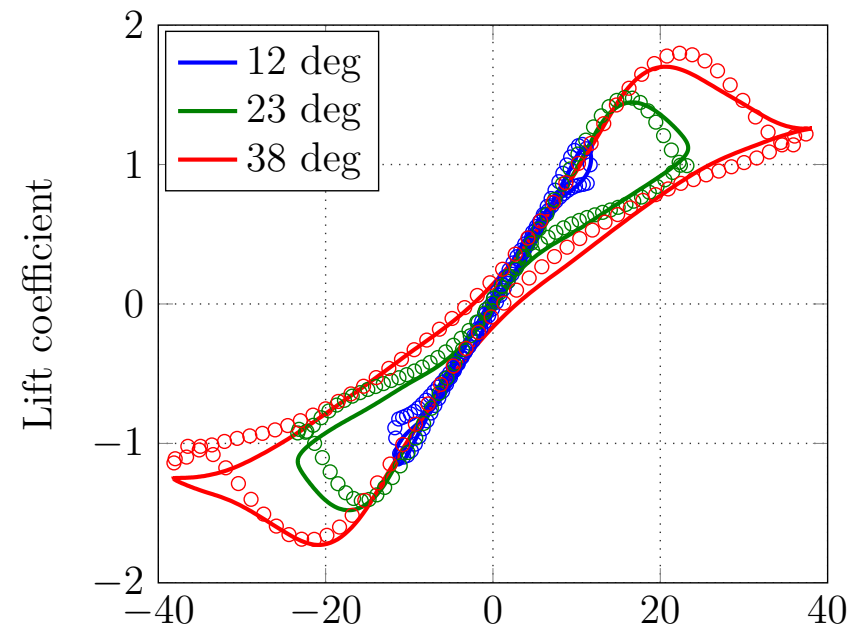

(a) Angle of attack (deg)

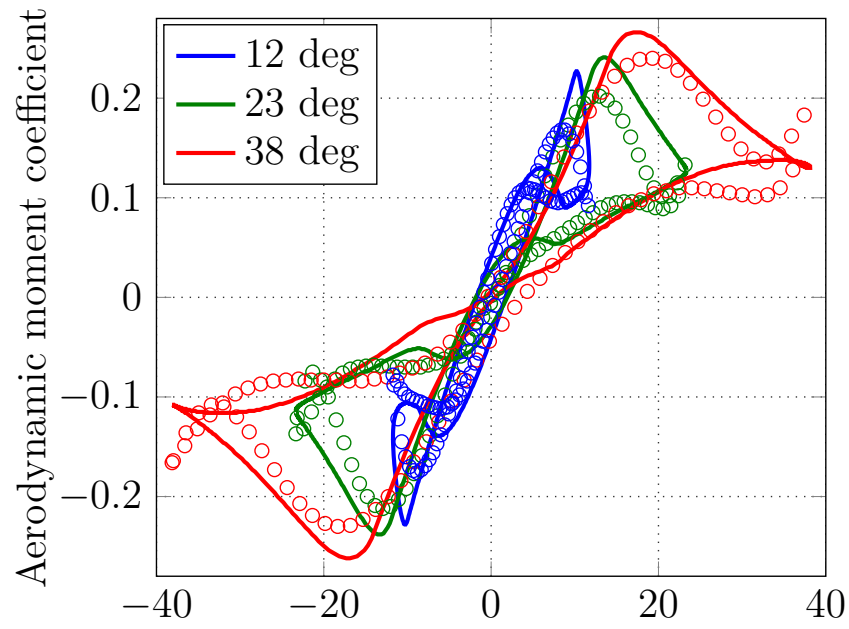

(b) Angle of attack (deg)

Figure 4: Lift (a) and Aerodynamic moment (b) coefficients measurements for 3 pitch amplitudes $\left(12^{\circ}\right.$ in blue, $23^{\circ}$ in green and $37^{\circ}$ in red). Circles are for measurements and solid lines for ONERA model estimation. The reduced frequency $2 \pi f U / b$ is equal to 0.03 and the Reynolds number to $2.210^{4}$. The ONERA parameters used for the model are reported in Table 1. 


\begin{tabular}{ccccccccc}
\hline \multicolumn{8}{c}{ Lift parameters } \\
\hline$\lambda^{L}$ & $\kappa^{L}$ & $\sigma_{0}^{L}$ & $r_{0}^{L}$ & $a_{0}^{L}$ & $\sigma_{2}^{L}$ & $r_{2}^{L}$ & $a_{2}^{L}$ & $E_{2}^{L}$ \\
\hline 0.119 & 0.81 & 0.1 & 0.15 & 0.24 & -0.005 & 0.09 & 0.26 & -0.004 \\
\hline \multicolumn{7}{c}{ Aerodynamic moment parameters } \\
\hline$\lambda^{M}$ & $\kappa^{M}$ & $\sigma_{0}^{M}$ & $r_{0}^{M}$ & $a_{0}^{M}$ & $\sigma_{2}^{M}$ & $r_{2}^{M}$ & $a_{2}^{M}$ & $E_{2}^{M}$ \\
\hline 0.1 & 0.43 & 0.15 & 0.19 & 0.4 & -0.026 & 0 & 0.08 & 0
\end{tabular}

Table 1: ONERA parameters corresponding to the dynamic stall measurements shown in figure 4. 


\section{Hysteretic behaviour of SMA}

The passive control strategy proposed in this paper is to use Shape Memory Alloys (SMA) springs in their pseudo-elastic regime as hysteretic dampers. Indeed when these alloys are subjected to cyclic deformations, they exhibit an hysteretic response allowing an important amount of energy dissipation. This hysteretic behaviour stems from a solid-solid phase change between two different states. The first one called austenite is stable at large temperatures and is the natural state of the spring at rest. The second state, present when the SMA is deformed, is called martensite, which is energetically stable at small temperatures and for which the microstructure is oriented (Delaey et al. (1974)). The energy dissipated during a cycle is equal to the area of the hysteresis loop so that the more the loop area is large, the more energy is prone to be dissipated in the device.

\subsection{Modelization of the hysteretic behaviour.}

The nonlinear behaviour of single dof SMAs can be derived from a general, three-dimensional model inferred from thermodynamical laws and then reduced by considering ad-hoc assumptions, see e.g. Bernardini and Vestroni (2003), Lacarbonara et al. (2004), D. Lagoudas et al. (2012), Ould Moussa (2012), Ould Moussa et al. (2012), and Popov and D. C. Lagoudas (2007). In this case the model contains, in-built within the oscillator equation, additional equations governing the evolution of the fraction of martensite, the description of heat transfer, and the thermodynamic force, the expression of which is derived from a pseudopotential of dissipation that can include yields functions in order to express the phase transformations, as explained by e.g. Ould Moussa et al. (2012). These modeling features are typical of hysteretic systems (Visintin (1994)) and give rise to a complex formulation which, in turn, induces numerical difficulties for solving the whole system.

Contrary to this approach, a heuristic model is used in this paper. Indeed, because the aim is to reproduce the global effect of the the SMA spring in pseudoelastic regime, the SMA can be seen as an hysteretic damper. A large variety of hysteresis model exist in the literature, e.g. the Dahl model (Dahl (1976)), the Duhem model (Duhem (1897) and Ivshin and Pence (1994)) or the one used in this paper, the Bouc-Wen model (Bouc (1971) and Wen (1976)). The latter has already been used to express the global restoring force of an hysteretic damper, see e.g. Carpineto et al. (2014) or Song and Der Kiureghian (2006). The retained formulation of the SMA spring restoring force $F_{N L}^{S M A}$ resulting from the Bouc-Wen model reads

$$
F_{N L}^{S M A}(h, t)=K_{E} h+K_{3} h^{3}+z(t),
$$

where $K_{E}$ describes the global stiffness of the material and $K_{3}$ the potential hardening or softening behaviour for large deformation. The hysteretic behaviour is modeled using the function $z$ which 


\begin{tabular}{c|c} 
Parameter & Definition \\
\hline$l_{0}$ & Spring length at rest \\
$l_{\text {ini }}$ & Spring length after a pre-strain \\
$\delta l$ & Amplitude step for a quasi-static loading \\
$\Delta l$ & Amplitude of one displacement cycle \\
$l_{f}$ & Displacement imposed to the spring \\
$f_{e}$ & Excitation frequency for a dynamic loading \\
$\epsilon_{i n i}$ & $l_{\text {ini }} / l_{0}$ \\
$\Delta \epsilon$ & $\Delta l / l_{0}$ \\
$\delta \epsilon$ & $\delta l / l_{0}$ \\
$\epsilon_{f}$ & $l_{f} / l_{0}$
\end{tabular}

Table 2: Definitions of the parameters used in the tensile tests experiments.

is estimated by means of this first-order differential equation,

$$
\dot{z}=\left[K_{D}-|z|^{n}(\gamma+\beta \operatorname{sign}(\dot{h} z))\right] \dot{h} .
$$

This equation depends on two kinds of parameters : $K_{D}, K_{E}$ and $K_{3}$ which contributes to the global linear and nonlinear stiffness of the material and $\beta, \gamma$ and $n$, the aim of which is to model the shape of the hysteresis loop. Eventually, $\operatorname{sign}()$ denotes the sign function. Because the model used is heuristic, $K_{D}, K_{E}, K_{3}, \beta, \gamma$ and $n$ have to be fitted experimentally by means of tensile tests.

\subsection{Tensile tests and model fitting}

In order to validate the use of the Bouc-Wen model, several tensile tests have been performed. The length of the spring at rest is called $l_{0}$. For all the experiments presented, the spring is prestrained by imposing a static length of deformation $l_{i n i}$ and then deformed periodically, with a peak-to-peak amplitude of loading $\Delta l$, around this pre-strained state. Besides we call $\epsilon_{i n i}$ (resp. $\Delta \epsilon$ ) the pre-strained rate (resp. peak to peak amplitude of loading rate). Eventually, $\epsilon_{f}$ is the displacement rate imposed to the spring, it can be quasi-static or dynamic as detailed below. These quantities are depicted in Fig. 5(b) and summarized in Table 2.

A first measurement set is realized with a quasi-static loading. A discrete displacement, with an amplitude step $\delta \epsilon$, is enforced to the spring and the restoring force is measured by a balance. An example of such measurement is shown in Fig. 5(a) for a given $\epsilon_{i n i}$ and several $\Delta \epsilon$, here $\delta \epsilon$ is equal to 0.1. In order to characterize the damping ability of the SMA we introduce an equivalent 
damping ratio $\zeta_{S M A}$ inspired from Carpineto et al. (2014) which reads (see Fig. 5(a))

$$
\zeta_{S M A}=\frac{\mathcal{A}_{S M A}}{K_{G}(\Delta l)^{2}}
$$

where $\mathcal{A}_{S M A}$ is the hysteresis loop area of the SMA spring and $K_{G}$ its global stiffness. These quantities are detailed in Fig. 5(a). The influence of $\epsilon_{i n i}$ and $\Delta \epsilon$ on $\zeta_{S M A}$ is shown in Fig. 5(c). Whereas $\epsilon_{i n i}$ has a little effect on the damping ratio $\zeta_{S M A}, \Delta \epsilon$ is directly proportional to the damping capacity. Hence, when used in a flutter experiment, one should try to maximize $\Delta \epsilon$.

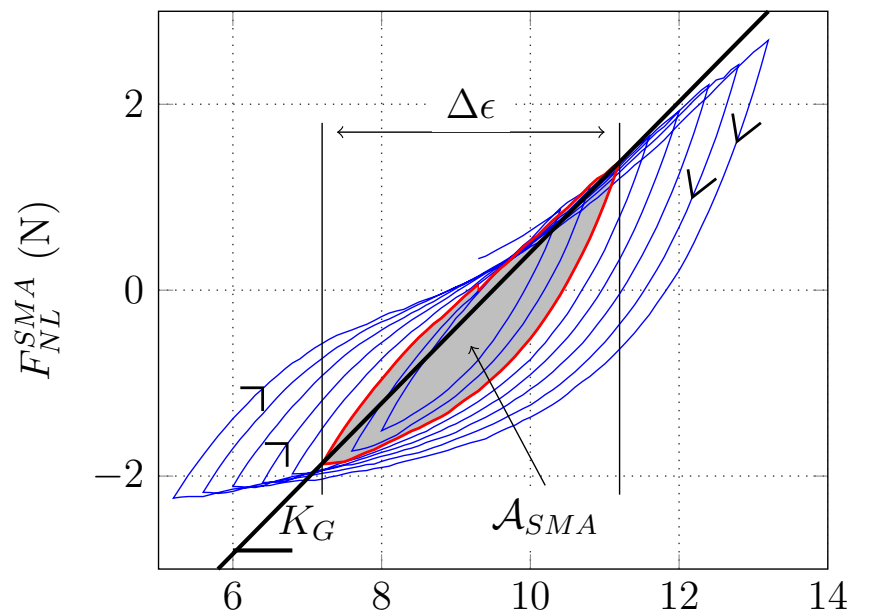

(a) $\epsilon_{f}$
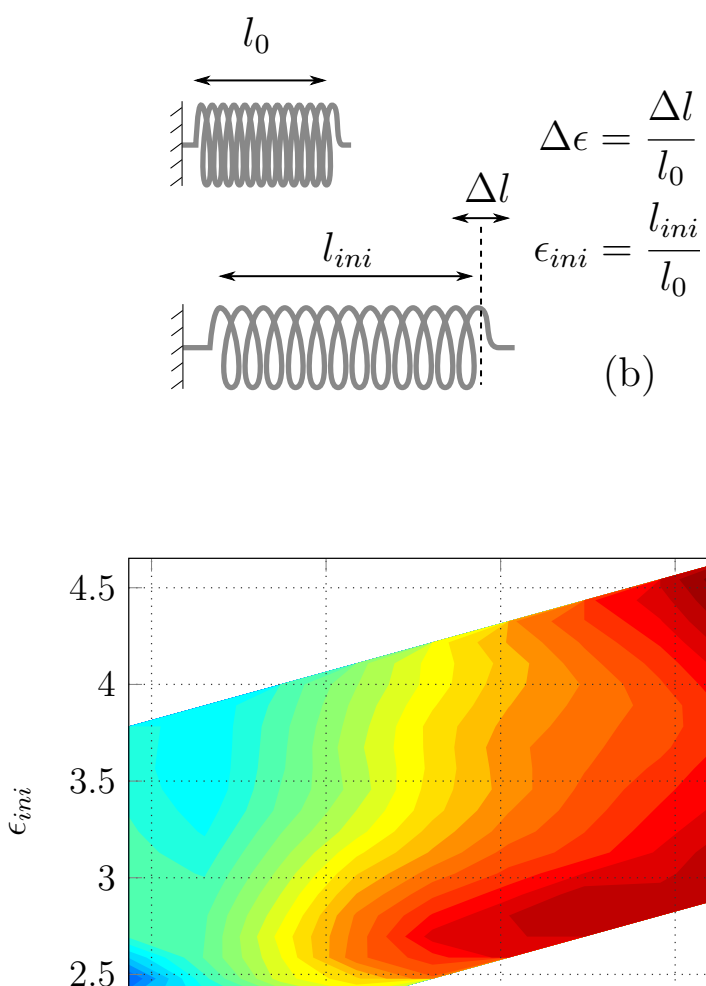

Figure 5: Quasi-static tensile test of SMA spring. (a) Example of quasi-static restoring force
for several amplitudes, $\delta \epsilon=0.1$ (b) Definition of spring quantities and (c) Cartography of SMA damping ratio vs strain rate per cycle and prestressing. 
A second measurement set is realized with a dynamic loading. In this case, the imposed displacement is $\epsilon_{f}(t)=\epsilon_{i n i}+(\Delta \epsilon / 2) \sin \left(2 \pi f_{e} t\right)$, where $f_{e}$ is the excitation frequency. This displacement is imposed by a slider-crank mechanism and the restoring force is measured by means of a force sensor. An example of such measurement is shown in Fig. 6(a) for $f_{e}=5 \mathrm{~Hz}$, which is in the range of frequency observed in the flutter experiment. For comparison, the quasi-static measurement obtained with the same values for $\epsilon_{i n i}$ and $\Delta \epsilon$ is also represented in Fig. 6(a). Even if the hysteresis loop decreases significantly in the dynamic case, the hysteresis phenomena is still present, and the damping effect of the SMA spring does not vanish. The evolution of the damping ratio as a function of $f_{e}$ is shown in Fig. 6(b). For small frequencies, $\zeta_{S M A}$ decreases significantly, nevertheless, after a certain frequency threshold $(\sim 0.3 \mathrm{~Hz}$ in the presented case), the damping ratio remains stable. The evolution of the damping capacity of SMAs has already been investigated in the past, see e.g Doaré et al. (2012), Yin et al. (2014) and Soul et al. (2010). These studies clearly evidenced that an optimum of damping capacity is obtained for a given excitation frequency, which has to be small, considering the physical processes involved. In the present case, this optimal frequency is clearly below the smallest tested frequency in Fig. 6(b).

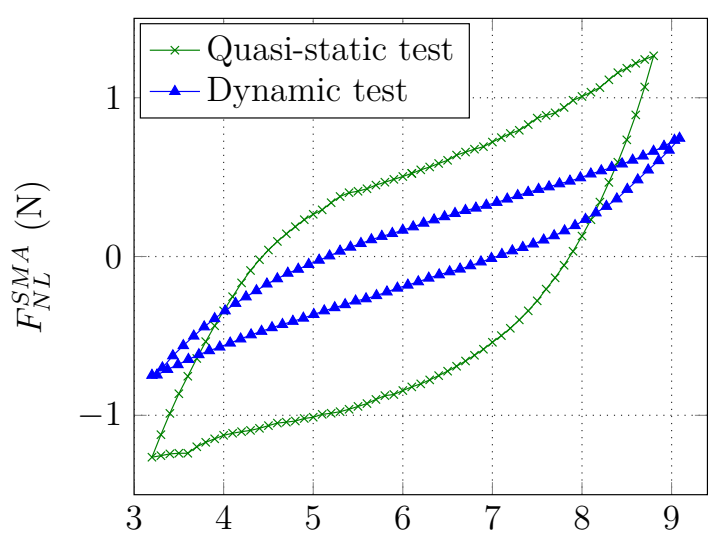

(a) $\epsilon_{f}$

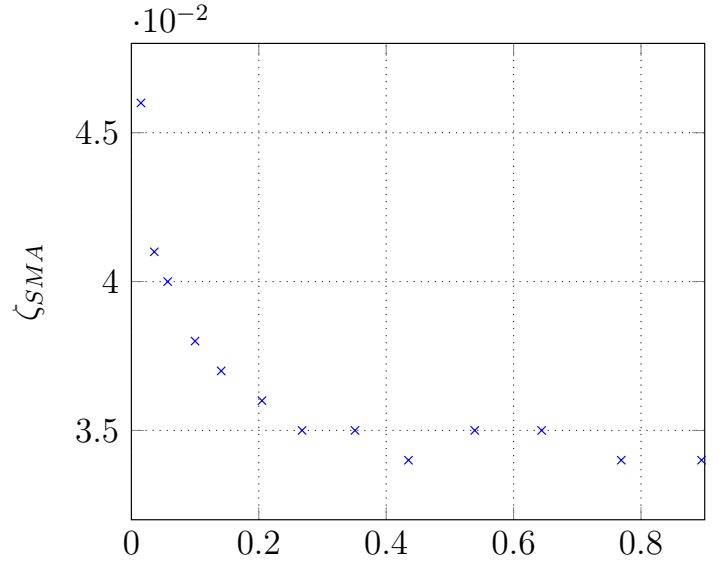

(b) $f_{e}$

Figure 6: SMA spring dynamic tensile test. (a) Comparison between quasi-static and dynamic tensile test and (b) influence of the excitation frequency on the SMA damping ratio.

The Matlab function fmincon is used to fit the Bouc-Wen model parameters $\left(K_{D}, K_{E}, K_{3}\right.$, $\beta, \gamma$ and $n$ ), the implemented procedure relies on a constrained nonlinear optimization. The objective function to minimize is the sum of the squared difference between measurements and model predictions. An example is presented in Fig. 7, where experimental measurements of the SMA spring restoring force with quasi-stating loading at several amplitudes are plotted with the result of the Bouc-Wen model with parameters from Table 3. One can observe the good agreement 


\begin{tabular}{ccc|ccc}
\multicolumn{2}{c|}{ Stiffness parameters } & \multicolumn{3}{c}{ Hysteresis parameters } \\
\hline$K_{D}\left[k g . s^{-2}\right]$ & $K_{E}\left[k g . s^{-2}\right]$ & $K_{3}\left[k g . m^{-2} \cdot s^{-2}\right]$ & $\beta$ & $\gamma$ & $n$ \\
\hline 138 & 0 & $8.710^{3}$ & 154 & 0 & 1
\end{tabular}

Table 3: Bouc Wen parameters corresponding to the case presented in Fig. 7

between model and experiments. It is remarked to the reader's attention, that the Bouc-Wen model parameters depend on the excitation frequency.

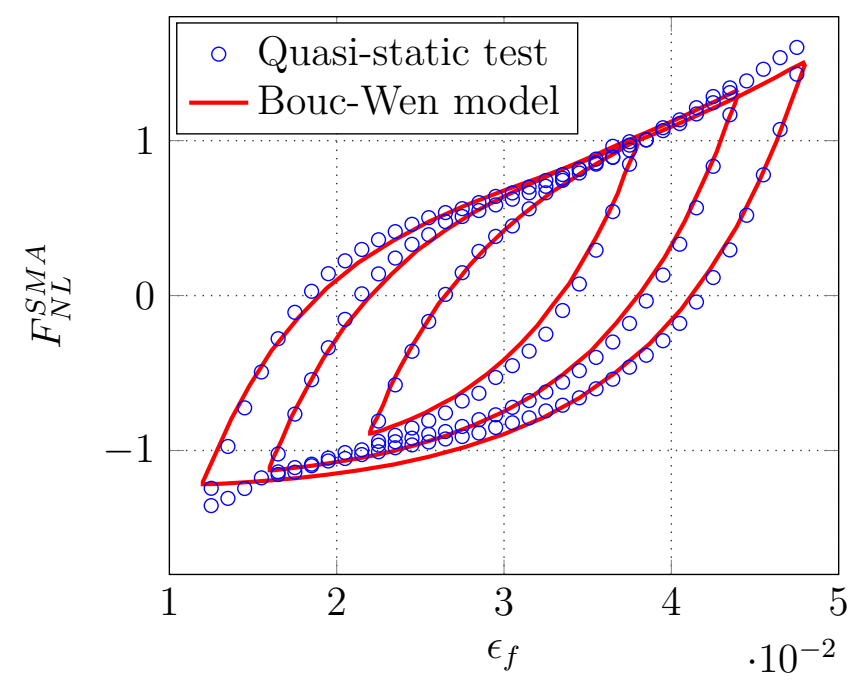

Figure 7: Comparison between SMA quasi-static tensile test for $\Delta \epsilon=1.6,2.4$ and 3.6 and BoucWen model estimation. 


\section{Experimental demonstration of flutter mitigation with SMA springs}

In this section, the experimental set-up used to demonstrate the effect of SMA on the flutter instability is presented. A typical result is shown on the post-critical regime of flutter with and without SMA.

\subsection{Flutter experimental set-up}

The flutter experiment is realized with a rectangular flat plate of span $225 \mathrm{~mm}$, chord $35 \mathrm{~mm}$ and thickness $1.5 \mathrm{~mm}$, which means a $4.3 \%$ thickness-to-chord ratio, the same ratio as the one used in section 2 for the dynamic stall measurements. The set-up is shown in Fig. 8, the flat plate is held with tow rods slotted into bearings, the pitch stiffness is carried out with spiral springs and the plunge stiffness with coil springs. The plunge spring restoring force is measured by means of force sensor PCB 208-C03 and the displacement of the two degrees of freedom with laser displacement sensors Keyence LK-G. The sensors are placed as shown in Fig. 8. To apply the control, the coil springs are replaced by the SMA springs with the appropriate pre-stress in order to have the same linear stiffness at small amplitude than the coil spring. The center of mass can be set by adding weights on the restoring bar (see Fig. 8). All the structural parameters are estimated by means of free decay tests. End plates are fastened to the flat plate ends to reduce 3D aerodynamic effects.
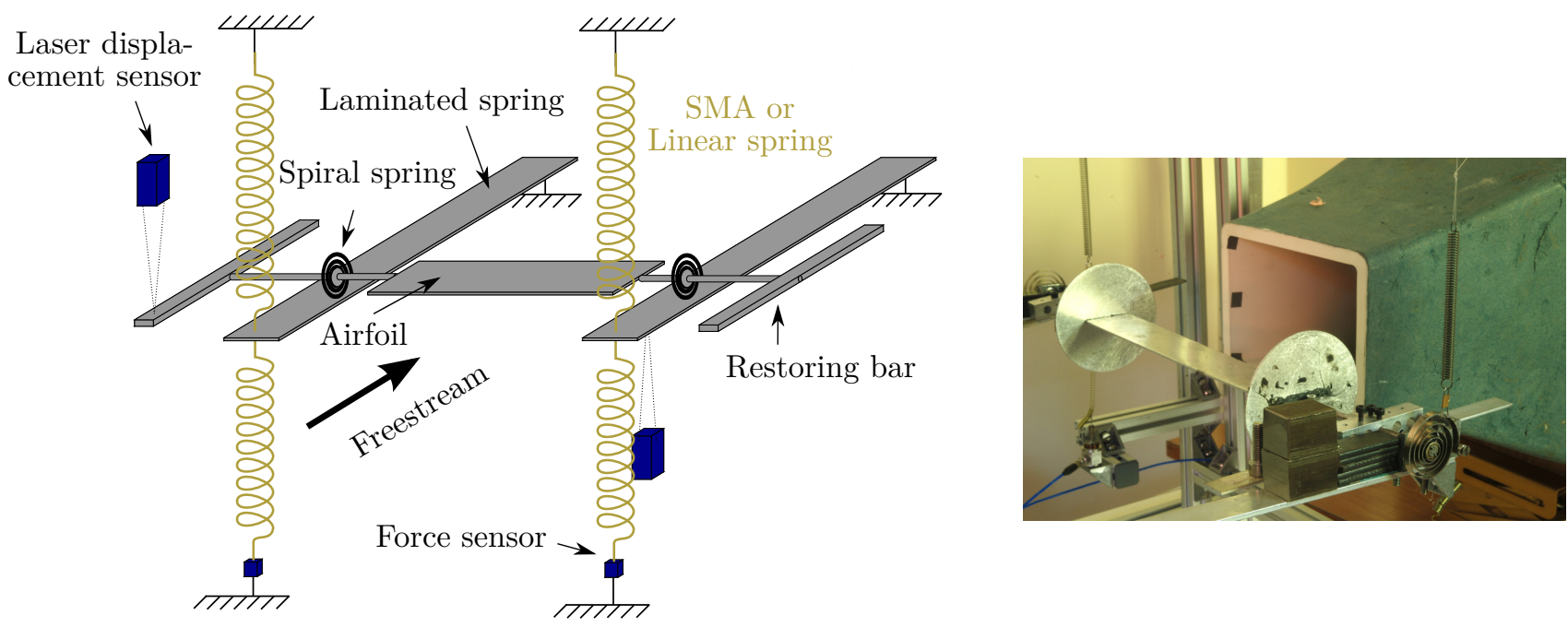

Figure 8: Sketch (left) and picture (right) of the experimental set-up used to study the flutter instability. 


\subsection{Flutter measurements}

An example of time response to an arbitrary initial condition with and without SMA springs is shown in Fig. 9(a). The flow velocity is equal to $9 \mathrm{~m} / \mathrm{s}$, which is beyond the flutter velocity calculated at $5.6 \mathrm{~m} / \mathrm{s}$. The corresponding restoring force of the plunge springs is shown in Fig. 9(b). The effect of using SMA springs is evidenced in Fig. 9(a), where the amplitude ratio between the limit cycles with and without SMA is equal to 2.53. Note that for the purpose of accurate comparison, the equivalent linear stiffness of the SMA is equal to that of the linear springs, as shown in Fig. 9(b) ; the only difference being the hysteresis loop of the strain-stress curve.

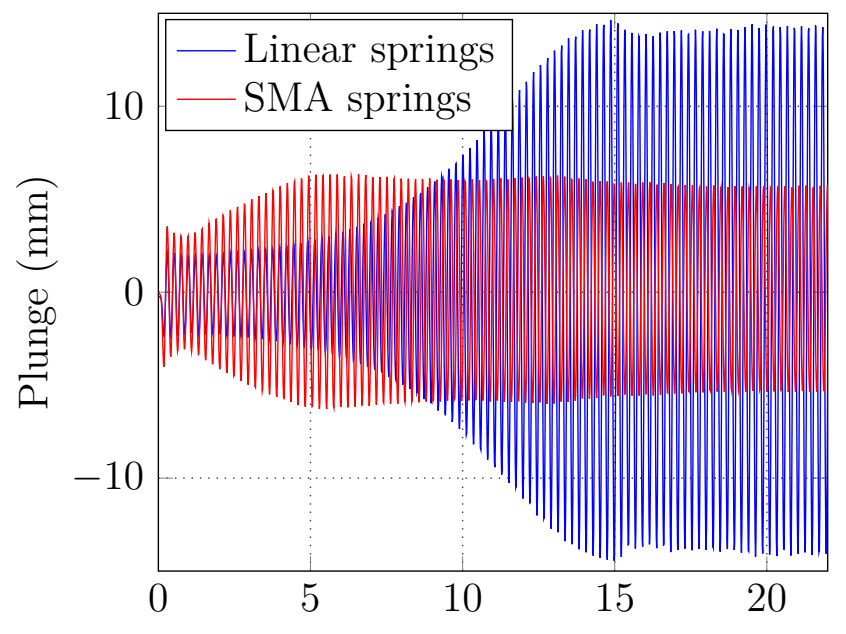

(a) Time $(\mathrm{s})$
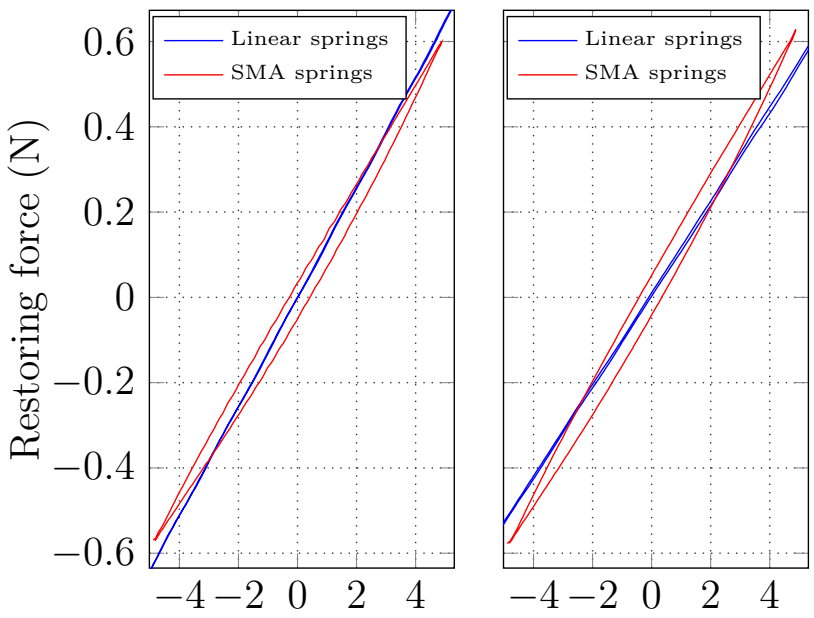

(b) Displacement $(\mathrm{mm})$

Figure 9: Time response measurement realized on the flutter experiment with and without SMA springs for $\mathrm{U}=9 \mathrm{~m} / \mathrm{s}$. (a) Displacement amplitude of the plunge mode. (b) Restoring force of the plunge springs on a stable LCO, the left plot is for left spring and the right plot for the right spring of the set-up (see Fig. 8).

As dry friction cannot be avoided in the bearings of the experimental set-up, the system does not encounter flutter oscillations near the flutter velocity in the absence of external perturbations. For that reason, a small disturbance is given to the flat plate when running the experiments. Consequently initial transient regimes are different from one experiment to the other. Nevertheless, repeated experiments have shown that, as in Fig. 9(a), the LCO settles down earlier with the SMA springs (at $\sim 5 \mathrm{~s}$ ) than with the linear springs $(\sim 15 \mathrm{~s})$. Hence the dissipated energy (by means of SMA) also helps in reducing the transient regime, which is logical since the damping capacity has been increased. Consequently, if the SMA springs are adjusted to dissipate energy before the airfoil encounters stall flutter, we can expect to prevent the dynamic stall phenomenon. This point will be addressed in section 5 . 
We focus now on the behaviour of LCOs for varying flow speeds, in order to draw a more complete flutter bifurcation diagram.

\subsection{Effect of SMA on flutter bifurcation diagram}

The time response measurement described previously is now realized for a range of flow speeds. The maximum flow speed value is set at $9 \mathrm{~m} / \mathrm{s}$, and then decreased step by step. At $9 \mathrm{~m} / \mathrm{s}$, a disturbance is applied to the plate so that the LCO can settle down. Then the amplitude of the LCO is recorded and the flow speed decreased. The resulting experimental bifurcation diagram is given in Fig. 10. The structural parameters identified for this measurement are reported in Table 4. Two main effects are observed. The first one is the reduction of LCO amplitude for all the flow speeds tested, one can observe a substantial gain in using SMA springs since the LCO amplitude has been divided by 3 on the plunge mode and by 1.6 the pitch mode, even if SMA springs are not applied to this degree of freedom. The second effect is the shift in the flow speed at which the instability disappears : with the SMA this flow speed is equal to $7 \mathrm{~m} / \mathrm{s}$ whereas without SMA springs it is equal to $5.6 \mathrm{~m} / \mathrm{s}$. These two results show that SMA springs are able to bring an important effect both on the amplitude of the LCO and on the size of the bifurcated branches. In order to analyze more deeply the post-flutter branches, a complete model including the aerodynamic forces from dynamic stall and SMA is investigated in the next section.

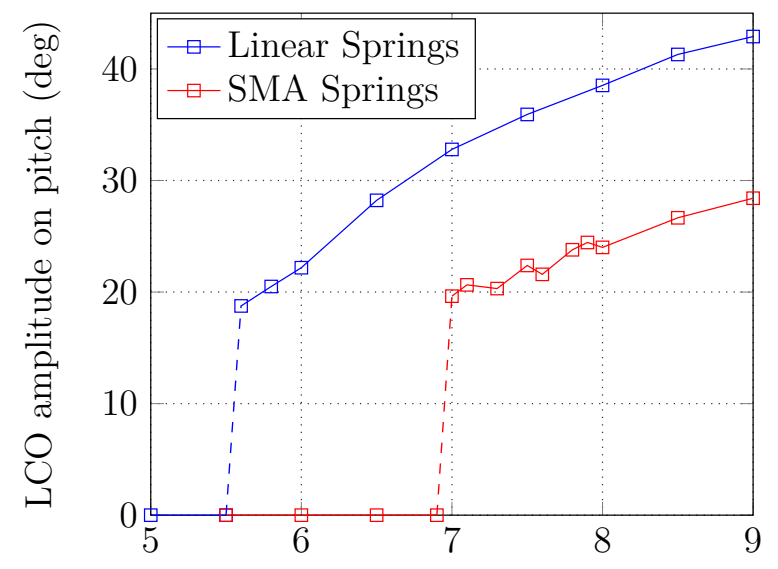

(a) Flow speed U (m/s)

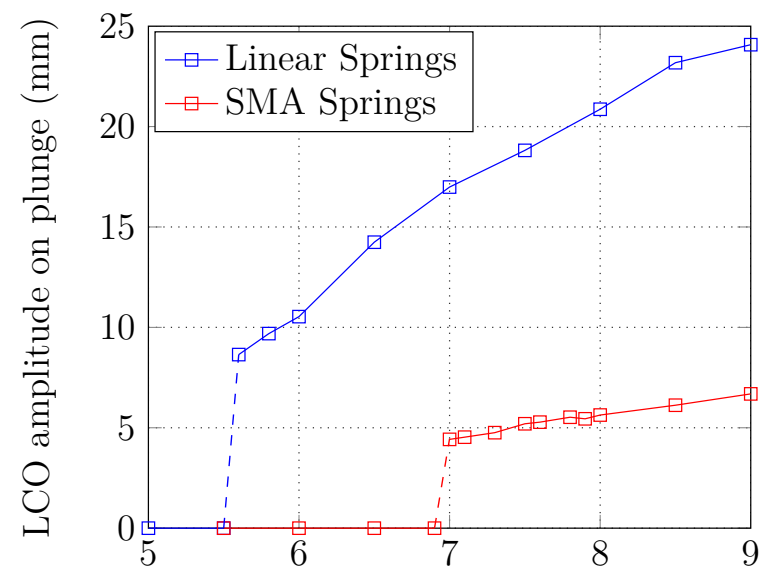

(b) Flow speed U (m/s)

Figure 10: Experimental measurement of LCO amplitude of pitch (a) and plunge (b) motion vs the flow speed $U$. 


\begin{tabular}{ccccccc}
$m[\mathrm{~kg}]$ & $I_{\alpha}\left[\mathrm{kg} \cdot \mathrm{m}^{2}\right]$ & $D_{h}\left[\mathrm{~kg} \cdot \mathrm{s}^{-1}\right]$ & $D_{\alpha}\left[\mathrm{kg} \cdot \mathrm{m}^{2} . \mathrm{s}^{-1}\right]$ & $K_{h}\left[\mathrm{~kg} \cdot \mathrm{s}^{-2}\right]$ & $K_{\alpha}\left[\mathrm{kg} \cdot \mathrm{m}^{2} \cdot \mathrm{s}^{-2}\right]$ & $S_{\alpha}[\mathrm{kg} \cdot \mathrm{m}]$ \\
\hline 0.389 & $2.11 \times 10^{-4}$ & 0.126 & $1.65 \times 10^{-4}$ & 282.3 & 0.143 & $1 \times 10^{-3}$
\end{tabular}

Table 4: Structural parameters of the flutter experiment presented in Fig. 10. 


\section{Influence of model parameters on the bifurcation diagram}

In this section, the aeroelastic, aerodynamic and SMA models are coupled in order to predict the evolution of the pitch and plunge motion numerically. The effect of the several parameters is detailed and the influence of the SMA springs is then discussed.

\subsection{Complete model}

The complete model including both effects of the nonlinear aerodynamic loads and the SMA nonlinear restoring forces reads :

$$
\begin{aligned}
& m \ddot{h}+S_{\alpha} \cos (\alpha) \ddot{\alpha}+\frac{b D_{h}}{U} \dot{h}+\frac{b^{2}}{U^{2}}\left(\delta_{l} K_{h}+\delta_{s} K_{E}\right) h-S_{\alpha} \sin (\alpha) \dot{\alpha}^{2}=-b^{3} \rho s\left(C_{l 1}+C_{l 2}\right)-\frac{\delta_{s} b^{2}}{U^{2}}\left(K_{3} h^{3}+z\right), \\
& I_{\alpha} \ddot{\alpha}+S_{\alpha} \cos (\alpha) \ddot{h}+\frac{b D_{\alpha}}{U} \dot{\alpha}+\frac{b^{2} K_{\alpha}}{U^{2}} \alpha=2 b^{4} \rho s\left(C_{m 1}+C_{m 2}\right) \\
& \dot{C}_{l 1}+\lambda^{L} C_{l 1}=\lambda^{L}\left(\left.\frac{\partial C_{l}}{\partial \alpha}\right|_{\alpha=0} W_{0}+\sigma^{L} W_{1}\right)+\left(\left.\kappa^{L} \frac{\partial C_{l}}{\partial \alpha}\right|_{\alpha=0}+d^{L}\right) \dot{W}_{0}+\kappa^{L} \sigma^{L} \dot{W}_{1} \\
& \ddot{C}_{l 2}+a^{L} \dot{C}_{l 2}+r^{L} C_{l 2}=-\left(\left.r^{L} \Delta C_{l}\right|_{W_{0} / U}+E^{L} \dot{W}_{0}\right), \\
& \dot{C}_{m 1}+\lambda^{M} C_{m 1}=\lambda^{M}\left(\left.\frac{\partial C_{m}}{\partial \alpha}\right|_{\alpha=0} W_{0}+\sigma^{M} W_{1}\right)+\left(\left.\kappa^{M} \frac{\partial C_{m}}{\partial \alpha}\right|_{\alpha=0}+d^{M}\right) \dot{W}_{0}+\kappa^{M} \sigma^{M} \dot{W}_{1} \\
& \ddot{C}_{m 2}+a^{M} \dot{C}_{m 2}+r^{M} C_{m 2}=-\left(\left.r^{M} \Delta C_{m}\right|_{W_{0} / U}+E^{M} \dot{W}_{0}\right) \\
& \dot{z}=\left[K^{D}-|z|^{n}(\gamma+\beta \operatorname{sign}(\dot{h} z))\right] \dot{h} .
\end{aligned}
$$

The time integration is realized using a 4th order Runge-Kutta scheme, at each time step the aerodynamics loads as well as the SMA restoring forces are computed and then introduced in the aeroelastic system in order to compute the value of $\alpha, \dot{\alpha}, h$ and $\dot{h}$ at the same time step. The parameters $\delta_{l}$ and $\delta_{s}$ introduced in equation (6a) are Kronecker-like delta symbols used to switch from one model to another. Indeed, the behaviour of the aeroelastic system without SMA springs is computed by selecting $\delta_{l}=1$ and $\delta_{s}=0$, whereas the motion with SMA springs is calculated by using $\delta_{s}=1$ and $\delta_{l}=0$. 


\subsection{Characteristics of the bifurcation diagram}

A typical result of the procedure presented above without SMA springs, i.e. with $\delta_{l}=1$ and $\delta_{s}=0$, is shown in Fig. 11 for the pitch motion. This generic result shows that the bifurcation diagram is composed of two branches with different amplitudes. The branch with a small amplitude, ranging from $U_{f}$ to $U_{s}$ on the forwarding branch, corresponds to classical flutter. The branch with a larger amplitude including the part of the forwarding branch ranging after $U_{s}$ and the part of the decreasing branch ranging after $U_{c}$, corresponds to stall flutter.

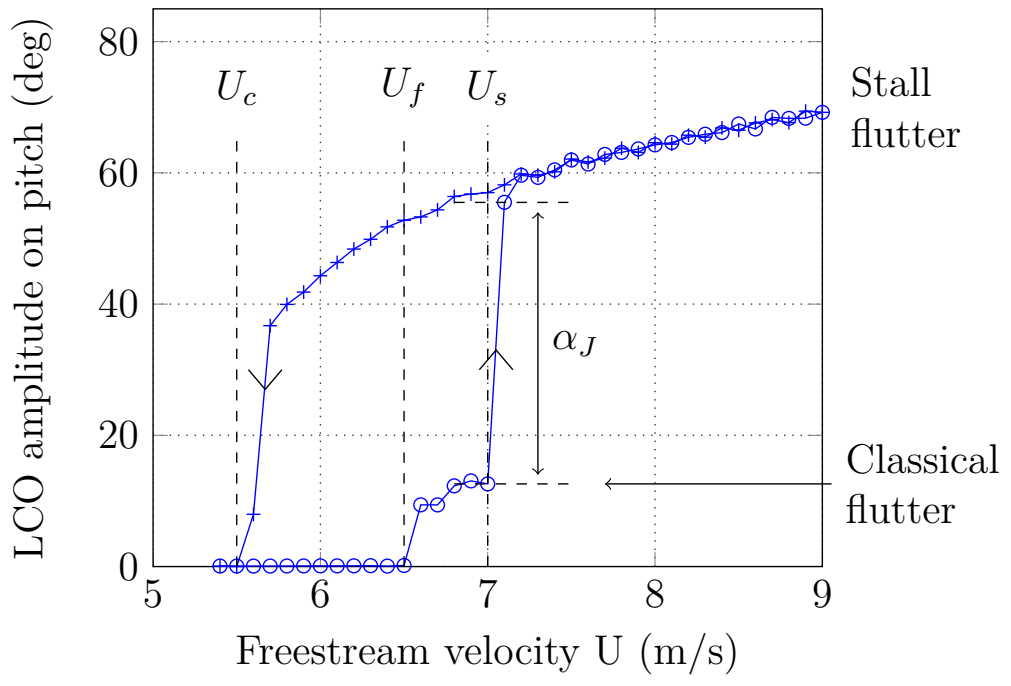

Figure 11: Amplitude of pitch LCO without SMA springs numerically calculated by resolving the complete system equation (6) for increasing [o] and decreasing $[+]$ flow speeds.

The bifurcation diagram can thus be completely analyzed by the following four characteristics : the three critical velocities $U_{c}, U_{f}$ and $U_{s}$; and the amplitude of the jump $\alpha_{J}$. The flutter velocity $U_{f}$ depends only on the structural parameters and on the lift and moment coefficient slope at zero angle of attack. Since the SMA springs are set such that their linear stiffnesses are equal to the linear spring stiffness at small amplitude, the SMA springs have no effect on this velocity. $U_{s}$ is the stall flutter speed. Stall flutter occurs when the angle of attack is larger than the airfoil stall angle, therefore the flow around the plate is entirely separated and reattached on the plate over one cycle of oscillation, see e.g. Razak et al. (2011). Increasing $U_{s}$ is very advantageous because the airfoil undergoes very large angle of attack when stall flutter appears. $U_{c}$ is the flow speed at which the instability stops, it is different from $U_{f}$ for a subcritical instability, which is detrimental for the structure. Eventually $\alpha_{J}$ is the amplitude gap in pitch for $U=U_{s}$, it is also a key quantity to prevent the structure from large deformations. $U_{s}, U_{c}$ and $\alpha_{J}$ rely on nonlinear phenomena, which 
means that the nonlinear behaviour of SMA springs might have an effect on them. To prevent the airfoil to encounter large deformations, the optimal control should :

- reduce $\alpha_{J}$,

- increase $U_{s}$,

- increase $U_{c}$ as much as possible; at best rendering the bifurcation supercritical.

Therefore, the ideal situation is to avoid stall flutter and keep the system in the classical flutter regime. The effect of aerodynamic forces on these quantities is discussed in section 5.3 and the influence of the SMA springs in section 5.5.

\subsection{Effect of aerodynamic forces}

Because the dynamic stall might be different from one airfoil to the other, an insight is given on the influence of the ONERA parameters on the bifurcation diagram of the 2 dofs system. With this aim in view, bifurcations diagrams have been calculated while varying each ONERA parameter in order to estimate its influence. The result of these investigations is summarized in Table 5 and illustrated in Fig. 12. For each ONERA parameter, the + signs indicate the qualitative magnitude of its effect on the bifurcation point.

\begin{tabular}{|c|c|c|c|c|c|c|c|c|c|}
\hline$O$ & \multicolumn{5}{|c|}{ Linear } & \multicolumn{4}{|c|}{ Nonlinear } \\
\hline Bif. pt & $\lambda^{L}$ & $\kappa^{L}$ & $\sigma_{0}^{L}$ & $r_{0}^{L}$ & $a_{0}^{L}$ & $\sigma_{2}^{L}$ & $r_{2}^{L}$ & $a_{2}^{L}$ & $E_{2}^{L}$ \\
\hline$U_{s}$ & + & +++ & $=$ & ++ & +++ & $=$ & $=$ & + & ++ \\
\hline$U_{c}$ & ++ & ++ & $=$ & ++ & +++ & ++ & ++ & + & +++ \\
\hline$\alpha_{J}$ & ++ & ++ & $=$ & +++ & +++ & +++ & +++ & ++ & ++ \\
\hline & $\lambda^{M}$ & $\kappa^{M}$ & $\sigma_{0}^{M}$ & $r_{0}^{M}$ & $a_{0}^{M}$ & $\sigma_{2}^{M}$ & $r_{2}^{M}$ & $a_{2}^{M}$ & $E_{2}^{M}$ \\
\hline$U_{s}$ & $=$ & +++ & $=$ & + & ++ & + & $=$ & $=$ & $=$ \\
\hline$U_{c}$ & + & +++ & $=$ & + & ++ & +++ & + & $=$ & +++ \\
\hline$\alpha_{J}$ & ++ & ++ & $=$ & + & ++ & +++ & + & $=$ & +++ \\
\hline
\end{tabular}

Table 5: Influence of the ONERA parameters on the identified points in the bifurcation diagram. The number of plus sign shows the magnitude of the influence on the bifurcation point, while an equal sign indicates that it has no effect. 
For a better understanding, four examples are highlighted in Fig. 12 where the blue curve is the bifurcation diagram presented in section 5.2. The examples have been chosen to cover the three categories depicted in section 2.2 and to show as much different situations as possible. In Fig. 12(a), the influence of $a_{0}^{L}$ is shown. By decreasing $a_{0}^{L}$ from 0.25 to 0.1 , it can be observed that the stall flutter is significantly postponed, $U_{s}$ is shifted from $7 \mathrm{~m} / \mathrm{s}$ to $7.7 \mathrm{~m} / \mathrm{s}$. Moreover the jump $\alpha_{J}$ goes down from 43 degrees to 6 degrees and the subcriticality is vanished, then $U_{c}=U_{f}$. As reported in Table $5, a_{0}^{L}$ is the parameter which has the most influence $(+++)$ on all bifurcations points. The influence of $a_{0}^{L}$ (and to a lesser extent of $a_{0}^{M}$ ) on the bifurcations points is large because this term appear as a damping coefficients in equation (6c) and is then more prone to have an effect on LCO amplitude. The influence of $\sigma_{2}^{M}$ is plotted in Fig. 12(b), its influence is substantial $(+++)$ on $\alpha_{J}$, moderate $(++)$ on $U_{c}$, and negligible $(=)$ on $U_{s}$. In comparison with $a_{0}^{L}$, the subcriticality is not canceled and the stall flutter is almost not shifted, and the main effect resides in the important decrease of the LCOs amplitude in stall flutter regime. Regarding $\kappa_{L}$, as shown in Fig. 12(c), this parameter has a large influence on $U_{s}(+++)$ but a moderate one $(++)$ on $\alpha_{J}$ and $U_{c}$. Eventually, $E_{2}^{M}$, shown in Fig. 12(d) has almost the same effect than $\sigma_{2}^{M}$.

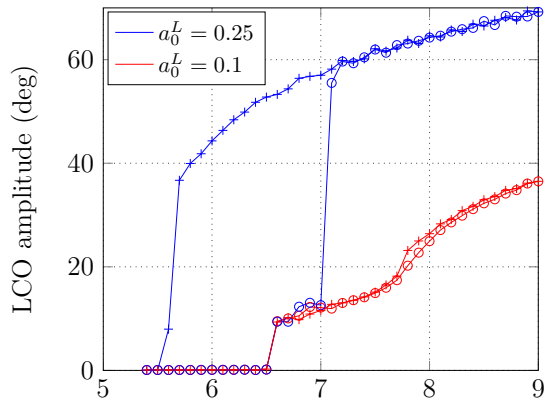

(a) Flow speed U (m/s)

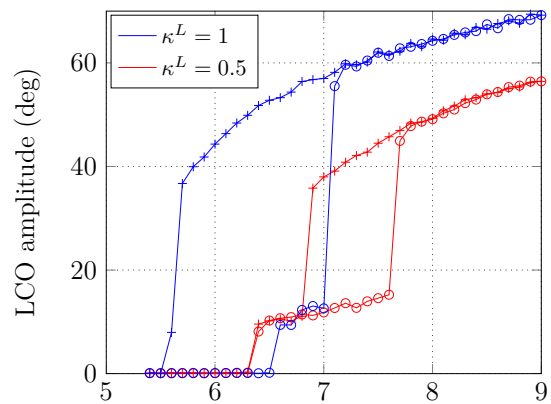

(c) Flow speed U (m/s)

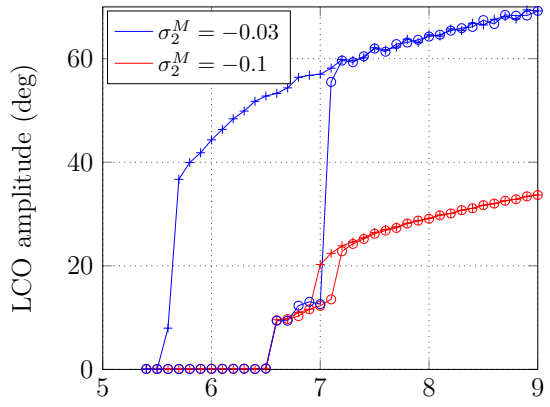

(b) Flow speed U (m/s)

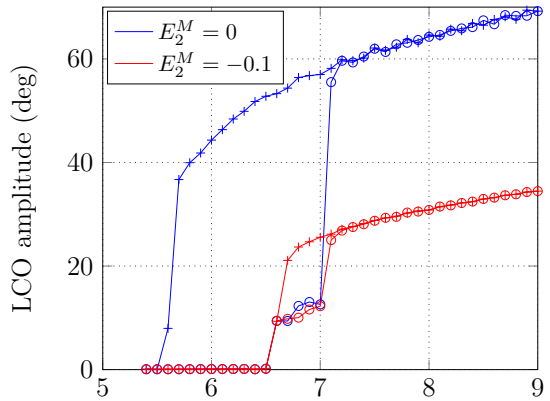

(d) Flow speed U $(\mathrm{m} / \mathrm{s})$

Figure 12: Influence of four ONERA parameters on the bifurcation diagram. The blue curve is for the reference case used in section 5.2 whereas the red curve is for the modified ONERA parameter. 


\begin{tabular}{ccccccccc}
$\lambda^{L}$ & $\kappa^{L}$ & $\sigma_{0}^{L}$ & $r_{0}^{L}$ & $a_{0}^{L}$ & $\sigma_{2}^{L}$ & $r_{2}^{L}$ & $a_{2}^{L}$ & $E_{2}^{L}$ \\
\hline 0.119 & 0.81 & 0.1 & 0.15 & 0.16 & -0.005 & 0.09 & 0.26 & -0.004 \\
\hline$\lambda^{M}$ & $\kappa^{M}$ & $\sigma_{0}^{M}$ & $r_{0}^{M}$ & $a_{0}^{M}$ & $\sigma_{2}^{M}$ & $r_{2}^{M}$ & $a_{2}^{M}$ & $E_{2}^{M}$ \\
\hline 0.1 & 0.43 & 0.15 & 0.19 & 0.4 & -0.026 & 0 & 0.08 & 0
\end{tabular}

Table 6: Fitted ONERA parameters used for the bifurcation diagram in Fig. 13.

\begin{tabular}{ccc|ccc}
\multicolumn{3}{c|}{ Stiffness parameters } & \multicolumn{3}{c}{ Hysteresis parameters } \\
\hline$K_{D}\left[\mathrm{~kg} \cdot \mathrm{s}^{-2}\right]$ & $K_{E}\left[\mathrm{~kg} \cdot \mathrm{s}^{-2}\right]$ & $K_{3}\left[\mathrm{~kg} \cdot \mathrm{m}^{-2} \cdot \mathrm{s}^{-2}\right]$ & $\beta$ & $\gamma$ & $n$ \\
\hline 141.15 & 141.15 & $1.710^{4}$ & 100 & 20 & 1.78
\end{tabular}

Table 7: Bouc-Wen parameters experimentally fitted used for the bifurcation diagram in Fig. 13.

\subsection{Model fitting to experiments}

The complete model presented in section 5.1 is now used to reproduce the experimental observations reported in section 4.3 .

For practical reasons, the flat plate used for the dynamic stall measurements and the one used for the flutter experiment are different, consequently the ONERA parameters are not the same in the two cases. Consequently we choose to fit the parameters of the ONERA model by a direct comparison with the bifurcation diagram; in order to highlight the versatility of the model. For that purpose the general results from Table 5 have been used as guidelines to make the bifurcation diagram of the model converge to the experimental one. The selected ONERA parameters are gathered in Table 6. On the other hand, the Bouc-Wen parameters have been directly fitted using the force measurements on the flutter set-up and are reported in Table 7. The corresponding bifurcation diagram is shown in Fig. 13. 


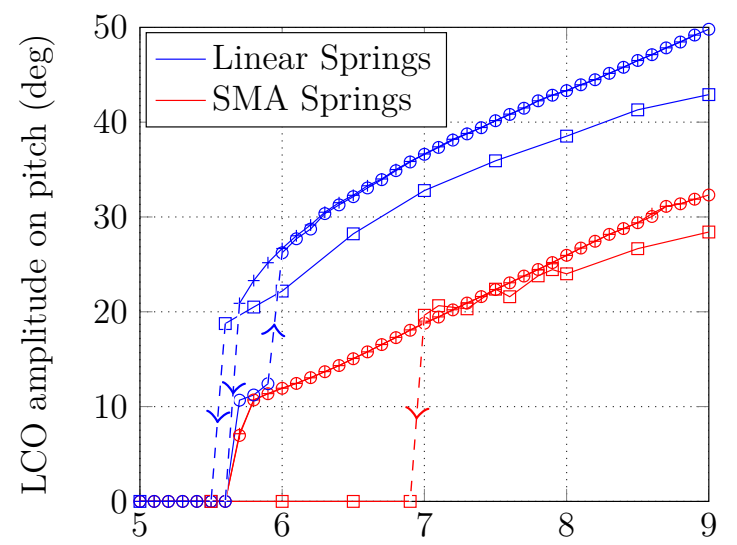

(a) Flow speed U (m/s)

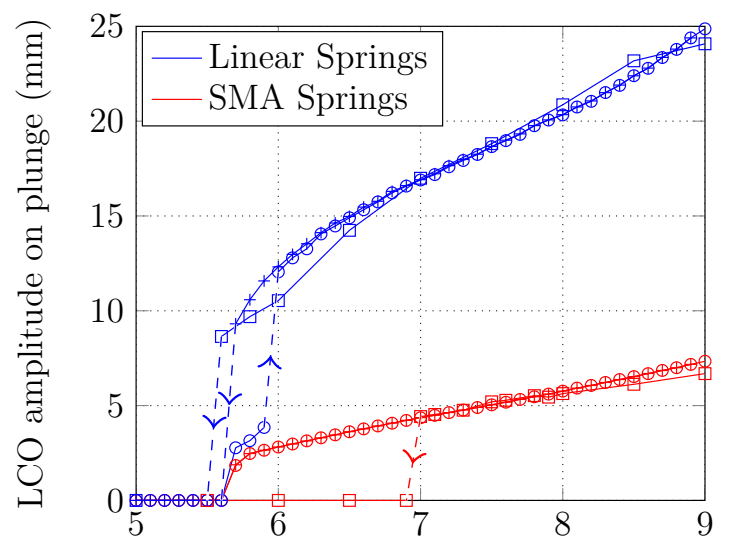

(b) Flow speed U (m/s)

Figure 13: LCO amplitude vs flow speed on pitch (a) and plunge (b) motion. Experimental results for decreasing flow speeds are denoted with squares, numerical calculations for increasing flow speeds are denoted with circles and for decreasing flow speeds with + signs.

The LCO amplitude is very well estimated on pitch and plunge, with and without SMA springs. The first main effect of SMA springs exhibited in section 4.3, which is the LCO amplitude reduction, is correctly reproduced by the numerical model. Indeed the ratio of LCO amplitude with and without SMA springs is equal to 1.70 numerically and 1.59 experimentally on pitch and to 3.58 numerically and 3.71 experimentally on plunge, which is very satisfactory. The second main effect of the SMA observed experimentally is the shift in $U_{c}$. It can be due either to the subcriticality of the flutter instability or to the fact that for $U$ close to $U_{c}$ the motion amplitude is relatively small (especially on plunge motion) and can then be stopped by the bearings dry friction before the instability really stops. Because the forwarding branch is not measured, the subcriticality of the instability cannot be properly established experimentally. Nevertheless, we observe numerically with several sets of ONERA and Bouc-Wen parameters that when the flutter instability is subcritical with linear springs it always becomes supercritical with SMA springs no matter what the value of the Bouc-Wen parameters. Thus $U_{c}$ can be shifted using SMA springs. Moreover, it is shown in Fig. 13, that the jump calculated numerically at $5.9 \mathrm{~m} / \mathrm{s}$ with linear springs is avoided with the SMA springs. Thus, the influence of SMA springs is close to the ideal situation presented in section 5.2.

\subsection{Effect of SMA springs on the flutter instability}

In this section, we discuss the influence of the hysteresis parameters of the Bouc-Wen model, $n$, $\gamma$ and $\beta$ on the flutter post-critical regime. One must keep in mind that the hysteretic behaviour can be realized by other device than SMA springs, see e.g Carboni and Lacarbonara, 2015; Carpineto et al., 2014, and that this study is still relevant since the hysteretic behaviour can be modeled with 
the Bouc-Wen model.

The effects of $n, \gamma$ and $\beta$ on the bifurcation diagram fitted with experimental measurements (Fig. 13) are exhibited in Fig. 14. We remark that their influence is mostly on the amplitude of the LCOs, since the presence of the SMA has canceled the jump and the subcriticality. Nevertheless each parameter does not affect both degree of freedom in the same way. Indeed, $\beta$ and $n$ have a larger effect on the plunge motion, whereas $\gamma$ has a larger influence on the pitch motion. Eventually, it can be deduced from Fig. 14 that the optimal control would be obtained with a large $\beta$ and a small $\gamma$ and $n$.
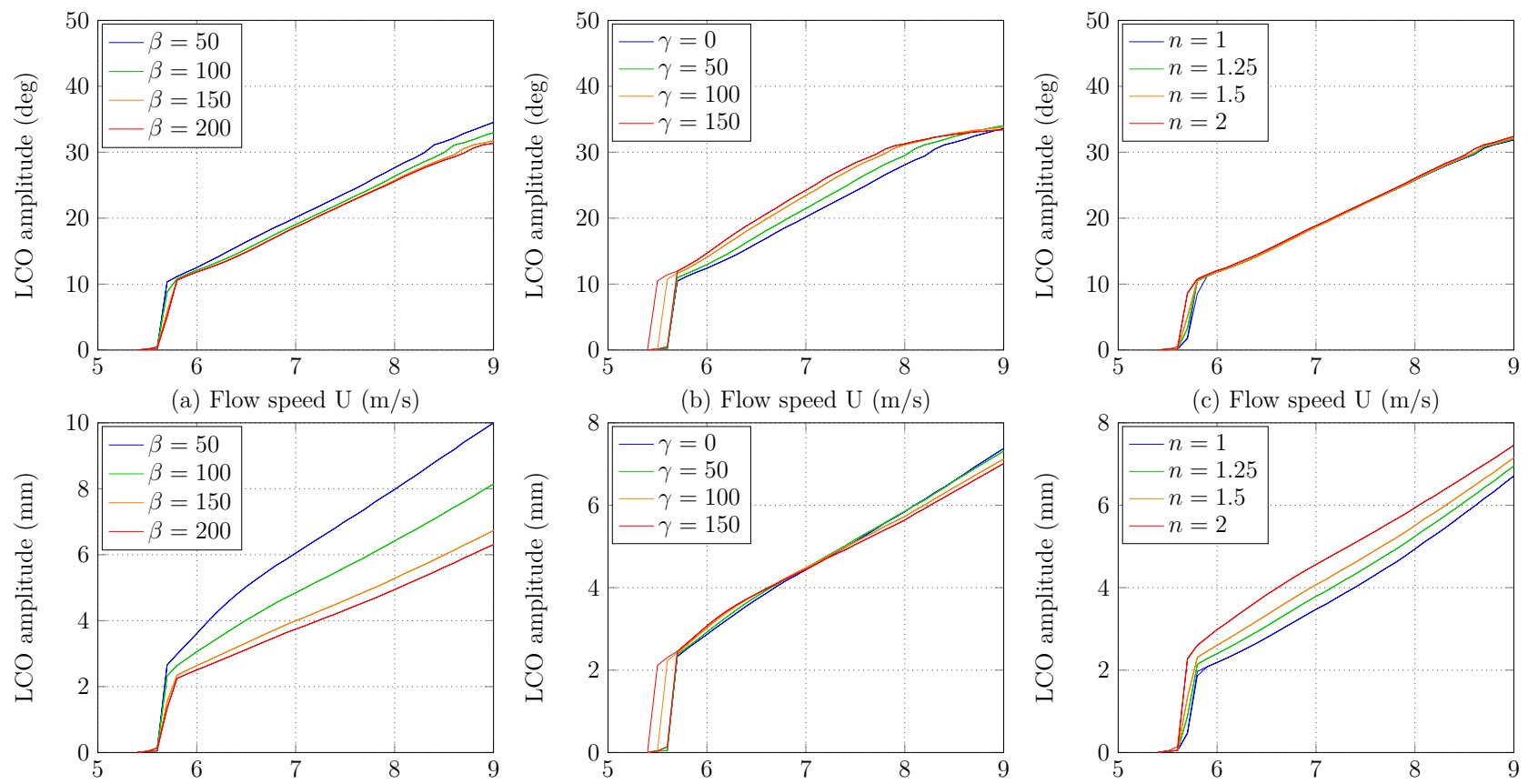

(d) Flow speed U (m/s)

(e) Flow speed U $(\mathrm{m} / \mathrm{s})$

(f) Flow speed U (m/s)

Figure 14: Influence of Bouc-Wen hysteretic parameters on the bifurcation diagram. First line : pitch motion; second line : plunge motion. (a,d) effect of $\beta$, (b,e) effect of $\gamma$ and (c,f) effect of $n$.

\section{Conclusion}

Whether one wants to harvest energy or avoid devastating instabilities, the control of aeroelastic flutter is a critical issue. An option is discussed here by using SMA springs in their pseudo-elastic regime, in order to use the potential of dissipation of such materials.

A numerical model has been proposed that takes into account the nonlinear aerodynamic forces along with the SMA springs nonlinear restoring force. An experiment including a flutter set-up with SMA springs has been conducted and show the efficiency of the latter to reduce the amplitude of the LCOs developing in the post-critical regime. 
The aeroelastic model has shown its capability to estimate a large variety of scenarios. Especially the influence of all the ONERA model parameters on the bifurcation caused by the flutter instability has been studied. Then the versatility of the ONERA model has been highlighted thanks to a model fitting to experimental bifurcations diagrams. Moreover, the Bouc-Wen model has shown its capability to reproduce the dynamic behaviour of the SMA springs as well as their influence on the flutter instability. A discussion on the role of each Bouc-Wen hysteresis parameters on the LCOs has been provided.

By means of the numerical model, it has been shown that the SMA springs always allow to cancel the jump at $U=U_{s}$ and the possible subcriticality of the instability. As a conclusion, this study shows undoubtedly the important effect that can be awaited by using hysteretic dampers for a passive control and mitigation of the flutter instability. Further research could extend the range of applications by considering e.g. vortex induced vibration or galloping instabilities.

\section{Acknowledgments}

We thanks Direction Générale de l'Armement (DGA) for a financial support to this project. 


\section{References}

Amandolèse, X., Michelin, S., and Choquel, M. 2013. Low speed flutter and limit cycle oscillations of a two-degree-of-freedom flat plate in a wind tunnel. Journal of Fluids and Structures 43, $244-255$.

Bernardini, D. and Vestroni, F. 2003. Non-isothermal oscillations of pseudoelastic devices. International Journal of Non-Linear Mechanics 38 (9), 1297-1313.

Bouc, R. 1971. Modèle mathématique d'hystérésis. Acustica 24 (3), 16-25.

Candido de Sousa, V. and De Marqui Junior, C. 2014. Effect of pseudoelastic hysteresis of shape memory alloy springs on the aeroelastic behavior of a typical airfoil section. Journal of Intelligent Material Systems and Structures 27 (1), 1-17.

Carboni, B. and Lacarbonara, W. 2015. Nonlinear dynamic characterization of a new hysteretic device: experiments and computations. Nonlinear Dynamics 83 (1-2), 23-39.

Carpineto, N., Lacarbonara, W., and Vestroni, F. 2014. Hysteretic tuned mass dampers for structural vibration mitigation. Journal of Sound and Vibration 333 (5), 1302-1318.

Dahl, P. R. 1976. Solid friction damping of mechanical vibrations. AIAA Journal 14 (12), 16751682.

Delaey, L., Krishnan, R. V., Tas, H., and Warlimont, H. 1974. Thermoelasticity, pseudoelasticity and the memory effects associated with martensitic transformations. Journal of Materials Science 9 (9), 1521-1535.

Doaré, O., Sbarra, A., Touzé, C., Ould Moussa, M., and Moumni, Z. 2012. Experimental analysis of the quasi-static and dynamic torsional behaviour of shape memory alloys. International Journal of Solids and Structures 49 (1), 32-42.

Dowell, E. H. 2004. A Modern Course in Aeroelasticity. Kluwer Academic Publishers.

Duhem, P. 1897. Die dauernden Anderungen und die Thermodynamik I. Z. Phys. Chemie 22 543589.

Ivshin, Y. and Pence, T. J. 1994. A constitutive model for hysteretic phase transition behavior. International Journal of Engineering Science 32 (4), 681-704.

Karpel, M. 1982. Design for active flutter suppression and gust alleviation using state-space aeroelastic modeling. Journal of Aircraft 19 (3), 221-227. 
Ko, J., Strganac, T. W., and Kurdila, A. J. 1999. Adaptive feedback linearization for the control of a typical wing section with structural nonlinearity. Nonlinear Dynamics 18 (3), 289-301.

Kwon, S.-D. and Park, K.-S. 2004. Suppression of bridge flutter using tuned mass dampers based on robust performance design. Journal of Wind Engineering and Industrial Aerodynamics 92 (11), 919-934.

Lacarbonara, W., Bernardini, D., and Vestroni, F. 2004. Nonlinear thermomechanical oscillations of shape-memory devices. International Journal of Solids and Structures 41 (5-6), 1209-1234.

Lacarbonara, W. and Cetraro, M. 2011. Flutter control of a lifting surface via visco-hysteretic vibration absorbers. International Journal Aeronautical and Space Sciences 12 (4), 331-345.

Lagoudas, D., Hartl, D., Chemisky, Y., Machado, L., and Popov, P. 2012. Constitutive model for the numerical analysis of phase transformation in polycrystalline shape memory alloys. International Journal of Plasticity 32 155-183.

Lee, B. H. K., Gong, L., and Wong, Y. S. 1997. Analysis and computation of nonlinear dynamic response of a two-degree-of-freedom system and its application in aeroelasticity. Journal of Fluids and Structures 11 (3), 225-246.

Lee, Y. S., Vakakis, A., Bergman, L., McFarland, D. M., and Kerschen, G. 2007a. Suppressing aeroelastic instability using broadband passive targeted energy transfers, part 1: theory. AIAA journal 45 (3), 693-711.

Lee, Y. S., Kerschen, G., McFarland, D. M., Hill, W. J., Nichkawde, C., Strganac, T. W., Bergman, L. A., and Vakakis, A. F. 2007b. Suppressing aeroelastic instability using broadband passive targeted energy transfers, part 2: experiments. AIAA journal 45 (10), 2391-2400.

Leishman, J. G. and Beddoes, T. S. 1989. A Semi-Empirical Model for Dynamic Stall. Journal of the American Helicopter Society 34 (3), 3-17.

Malher, A., Doaré, O., and Touzé, C. 2015a. Étude de l'influence d'un amortisseur pseudoélastique sur l'instabilité de flottement. CFM, Congrès Français de Mécanique.

- 2015. b. Pseudoelastic Shape Memory Alloys to Mitigate the Flutter Instability: A Numerical Study. Structural Nonlinear Dynamics and Diagnosis. Springer, 353-365.

Ould Moussa, M. 2012. Modélisation du comportement dynamique non-linéaire des structures en matériaux à mémoire de forme. Doctoral dissertation. Ecole Polytechnique. 
Ould Moussa, M., Moumni, Z., Doaré, O., Touzé, C., and Zaki, W. 2012. Non-linear dynamic thermomechanical behaviour of shape memory alloys. Journal of Intelligent Material Systems and Structures 23 (14), 1593-1611.

Petot, D. 1989. Modélisation du décrochage dynamique par équations différentielles. La Recherche Aérospatiale 5, 59-72.

Popov, P. and Lagoudas, D. C. 2007. A 3-D constitutive model for shape memory alloys incorporating pseudoelasticity and detwinning of self-accommodated martensite. International Journal of Plasticity 23 (10), 1679-1720.

Razak, N. A., Andrianne, T., and Dimitriadis, G. 2011. Flutter and stall flutter of a rectangular wing in a wind tunnel. AIAA journal 49 (10), 2258-2271.

Song, J. and Der Kiureghian, A. 2006. Generalized Bouc-Wen model for highly asymmetric hysteresis. Journal of engineering mechanics 132 (6), 610-618.

Soul, H., Isalgue, A., Yawny, A., Torra, V., and Lovey, F. C. 2010. Pseudoelastic fatigue of NiTi wires: frequency and size effects on damping capacity. Smart Materials and Structures 19 (8), 085006 .

Vipperman, J. S., Clark, R. L., Conner, M., and Dowell, E. H. 1998. Experimental active control of a typical section using a trailing-edge flap. Journal of Aircraft 35 (2), 224-229.

Visintin, A. 1994. Differential models of hysteresis. Springer Berlin.

Wen, Y. K. 1976. Method for random vibration of hysteretic systems. Journal of the engineering mechanics division 102 (2), 249-263.

Yin, H., He, Y., and Sun, Q. 2014. Effect of deformation frequency on temperature and stress oscillations in cyclic phase transition of NiTi shape memory alloy. Journal of the Mechanics and Physics of Solids 67, 100-128. 